\title{
Components of Emotion Dysregulation in Borderline Personality Disorder: A Review
}

\author{
Ryan W. Carpenter and \\ University of Missouri, Columbia, USA \\ Timothy J. Trull \\ University of Missouri, Columbia, USA, Department of Psychological Sciences, University of \\ Missouri, 210 McAlester Hall, Columbia, MO, USA
}

Timothy J. Trull: TrullT@missouri.edu

\begin{abstract}
Following Linehan's biosocial model, we conceptualize emotion dysregulation in borderline personality disorder (BPD) as consisting of four components: emotion sensitivity, heightened and labile negative affect, a deficit of appropriate regulation strategies, and a surplus of maladaptive regulation strategies. We review the evidence supporting each of these components. Given the complexity of the construct of emotion dysregulation and its involvement in many disorders, there is a need for research that specifies which components of emotion dysregulation are under study and also examines the interplay amongst these emotion dysregulation components.
\end{abstract}

\section{Keywords}

Borderline personality disorder; BPD; Emotion sensitivity; Biosocial theory; Affective instability; Negative affect; Emotion regulation; Distress tolerance; Emotion dysregulation; Emotion regulation strategies; Inadequate appropriate regulation strategies; Maladaptive regulation strategies; Psychiatry

\section{Introduction}

Borderline personality disorder is a serious mental disorder that is present in in $1-3 \%$ of the general population and, clinically, is the most commonly diagnosed personality disorder, present in $10 \%$ of patients in outpatient settings, $15-20 \%$ of patients in inpatients settings, and $30-60 \%$ of patients diagnosed with personality disorders $[1,2 \bullet, 3]$. Although often considered a 'disorder of dysregulation,' including dysregulation in the areas of interpersonal relationships, behavior, identity, and cognition, emotion dysregulation is considered to lie at the core of the disorder $[4,5 \bullet, 6,7]$.

Emotion dysregulation is the inability to flexibly respond to and manage emotions. Although this definition may appear straightforward, there is considerable variation in the phenomena studied under the heading of emotion dysregulation in BPD. Some researchers have focused on emotion sensitivity, others on affective intensity or affective lability, still others on emotional vulnerability, and so on. This is not especially surprising, given the complexity of

(C) Springer Science+Business Media New York 2012

Correspondence to: Timothy J. Trull, Trulltemissouri . edu.

Disclosure No potential conflicts of interest relevant to this article were reported. 
the construct. One way to understand these disparate approaches is to view emotion dysregulation as a process, incorporating multiple interactive components, and not as an end-state [8].

According to Linehan's biosocial theory $[4,5 \bullet]$, individuals with BPD are emotionally sensitive from birth. This sensitivity leads to a propensity to experience negative affect across contexts and situations, which then makes it difficult to learn appropriate emotion regulation strategies. This deficit in appropriate regulation strategies likely contributes to a tendency to engage in dysregulated behaviors in order to manage and reduce negative affect. This four component process results in negative consequences, which, in turn, reinforce emotion sensitivity. The result is a recursive pattern of emotion dysregulation (Fig. 1).

The present article takes this multi-component model of emotion dysregulation as its organizational framework. Instead of focusing on the broad construct of emotion dysregulation, we briefly review the evidence for each of the four components implicated in the biosocial model: Emotion sensitivity, heightened and labile negative affect, a deficit of appropriate regulation strategies, and a surplus of maladaptive regulation strategies. Note that although a consideration of these components provides the groundwork for a dynamic process model of emotion dysregulation, at present there is not enough empirical evidence to detail the interplay and direction of influence of these components. After reviewing recent studies targeting these components, we end this review with brief considerations for future research.

\section{Emotion Sensitivity}

Emotion sensitivity, the first component of our model, is thought to have biological origins and to be present from early life $[4,5 \cdot]$. It consists of a heightened emotional reactivity to environmental stimuli, including emotions of others. Emotion sensitivity in BPD has primarily been associated with negative mood states (e.g., anger, fear, sadness) and not positive emotions (although see $[9,10]$ ). Along these lines, Carlson, Egeland, and Sroufe [11] found, in a prospective longitudinal study of 162 individuals, that measures of negative emotionality, a construct presumably influenced by emotion sensitivity, in early childhood and adolescence predicted later BPD symptoms.

Many studies of emotion sensitivity in individuals with BPD have employed traditional laboratory-based cognitive tasks using emotional stimuli. For example, in the typical Stroop task [12], individuals are presented with words displayed in different colors and must ignore the word itself but name the color. In the emotional Stroop task, the words vary on their emotional significance. Theoretically, individuals sensitive to emotionally charged stimuli should have more difficultly suppressing their inclination to read the words, slowing their reaction times. Some studies have found that BPD individuals have slower reaction times on the emotional Stroop task $[9,10,13]$; however, others did not [14-16].

Although Wingenfeld et al. [16] did not find any significant reaction time differences between individuals with BPD and controls, except that the BPD group was slower overall, they found suggestive between-group differences in brain activity. Specifically, they found that the control group showed increased activation in the anterior cingulate cortex (ACC) and frontal lobe during the task, while the BPD group did not. This effect was particularly strong in controls when the negative stimuli were selected specifically to relate to a stressful event participants had self-reported before the experiment. The findings indicated that BPD individuals may have a cognitive, and especially attentional, bias toward processing negatively-valenced stimuli. 
Studies of emotion recognition in BPD individuals similarly suggest a negativity bias in emotion recognition (for a review, see [17]). That is, BPD individuals appear to be biased toward identifying negative emotions in others [18-21]. This is despite, or in conjunction with, a tendency to be less accurate in correctly identifying emotions in facial stimuli [2225]. The mixed findings concerning overall accuracy in identifying emotions may be due to the different types of stimuli used (e.g., social versus non-social), different instructions offered, and different emotions examined [26].

\section{Negative Affect}

The second component of emotion dysregulation in BPD is experiencing high levels of negative affect. This is theorized to be a direct consequence of emotion sensitivity, which, as stated above, has been primarily demonstrated to be specific to sensitivity toward negative mood states [27]. Findings suggest that negative affect is strongly associated with BPD [28, 29], in terms of both intensity and reactivity as assessed by self-report and psychophysiological measures [30]. Individuals with BPD appear to experience more negative affect, on average, than individuals without BPD [e.g., 22, 31•], but the evidence is somewhat inconsistent and differences are not always large [e.g., 27, 32, 33].

However, the most important characteristic of negative affect in BPD is perhaps not its absolute level, but its instability over time and, particularly, its ability to intensify rapidly and without much warning. This, too, is a likely result of emotion sensitivity, as the high level of reactivity to environmental stimuli in BPD individuals, especially toward events that are subtle or may seem innocuous to individuals without BPD, would be expected to cause rapid changes in mood.

The assessment of affective instability in BPD requires a more nuanced assessment approach than what traditional clinical assessment approaches (e.g., cross-sectional assessment, retrospective assessment) can provide, because affective instability is a dynamic, time-dependent process [34••]. Methods like ecological momentary assessment (EMA) [35], however, can provide multiple assessments of mood per day over many days [36] and reveal acute increases in negative affect from one moment or occasion to the next [33]. This high-frequency assessment approach yields "intensive longitudinal data" [37]. This is the only way to precisely assess the dynamics of mood states, extreme changes of mood, and environmental triggers for mood changes. Approaches that involve retrospective assessment of mood or mood changes are limited, as are traditional trait questionnaire approaches [38]. Individuals are notoriously poor historians, even for significant events and experiences. Asking individuals to aggregate experiences across time, as retrospective approaches do, is even more dubious (e.g., to rate themselves on the frequency and intensity of an unstable process, like affective instability).

An added advantage of EMA is that mood states are assessed in the person's natural habitat. In other words, we are able to obtain a more ecologically valid assessment of a person's mood in his or her daily life [39], while at the same time sampling experiences and events that may serve as antecedents, covariates, or consequences of mood changes [34••]. This focus on ecological validity differentiates daily life sampling methods like EMA from laboratory-based assessments of emotional dysregulation. For example, there are laboratory paradigms for eliciting emotional reactions (e.g., using pictures and films) and the individual's reactions can be assessed using brain imaging, coding of facial expression, or physiological response. However, in each case, it is unclear how such findings translate into real-world experiences.

EMA studies generally find greater instability of negative affect in BPD (for a review, see [31•]). Here, we discuss two more recent EMA studies of the instability of negative affect in 
BPD. Ebner-Priemer et al. [40] suggested that previous diary studies of affective instability in BPD had found mixed results because of the methods used by these studies to measure affective instability. They recruited 50 participants with BPD and 50 controls who carried an electronic diary with them for $24 \mathrm{hrs}$. Participants were prompted every 10-20 minutes to answer questions about their current emotional state. For their analyses, Ebner-Priemer et al. [40] identified three components of instability that needed to be taken into account:

Amplitude, whether changes are large or small, frequency, whether changes are rare or frequent, and temporal dependency, or in what sequence changes occur [41]. Previous analyses had largely overlooked amplitude and temporal dependency. Ebner et al. [40] used mean squared successive difference (MSSD) scores, which weight larger successive changes more and thereby account for amplitude and temporal dependency, and found that individuals with BPD were more unstable on scores of emotional valence and distress. They also found that BPD individuals were prone to large decreases in positive mood and that about half of these sudden decreases resulted in a negative mood state, compared to only 9 $\%$ of the declines in healthy controls.

Trull et al. [33] compared participants with BPD or major depressive disorder (MDD) over 28 days. Participants carried electronic diaries and reported on their positive and negative mood up to six times per day. Due to the multiple assessments for each individual per day, Trull et al. [33] used multilevel modeling to account for differences between groups of participants. BPD participants displayed more variability over time in both positive and negative affect. Using MSSDs adjusted for length of time interval between assessments, they found BPD individuals showed more instability of hostility, fear, and sadness, but not positive affect or general negative affect. Those with BPD were also more likely to report extreme changes for hostility scores. Interestingly, BPD individuals did not report greater mean levels of positive or negative emotions than MDD individuals. In sum, Trull et al. [33] extended the findings of Ebner-Priemer et al. [40], finding greater instability of specific negative affects over a longer period of time and in comparison to a clinical control group.

\section{Inadequate Emotion Regulation Strategies}

The third component of emotion dysregulation in BPD is a deficit in appropriate emotion regulation strategies. Negative affect is an inescapable part of everyday life for individuals with and without BPD. The majority of individuals without BPD are more or less able to manage their negative emotions most of the time with relative success. According to the biosocial theory, however, individuals with BPD, as emotionally-sensitive children experiencing heightened negative affect, did not learn the necessary skills to regulate emotion. As a result, they have difficulties with controlling what emotions they have, when they have them, and how those emotions are experienced [42]. Thus, strategies of emotion regulation can be targeted at shaping emotions before they occur or at modulating the emotion after it has begun [43]. We focus specifically on (lack of) adaptive strategies in this section, distinguishing them from maladaptive strategies, covered in the next section.

As already noted, the ability to identify what emotions one is experiencing is an important part of emotion regulation. Individuals with BPD have lower emotional awareness [44]. Similar to emotional awareness, is the ability to distinguish among emotional states, called emotional granularity [45]. Those high in emotional granularity are able to reliably and accurately differentiate their emotional states (e.g., distinguish sadness from anger). In contrast, those low in emotion granularity tend to describe emotional states in more global terms (e.g., feeling good or feeling bad). Suvak et al. [46•] recently evaluated emotional granularity in 46 individuals diagnosed with BPD, and compared their performance to that of 51 controls. Results indicated that BPD individuals scored significantly lower than controls on measures of emotional clarity, mood labeling, and emotional labeling focused on 
arousal versus valence. In the same vein, a recent EMA study found that BPD individuals experienced greater polarity (i.e., all-or-nothing) in their affect [47•]. These findings are consistent with clinical observations that those with BPD have more difficulty identifying, differentiating, and labeling emotions (especially if they are of the same valence).

In addition to recognizing and labeling their emotions, individuals with BPD have been found to have more problems employing emotion regulation strategies, based on both selfreport and behavioral measures [48-51], and neuroimaging studies [52, 53]. Low levels of distress tolerance [54] are also associated with BPD [50], suggesting that those with BPD have not developed coping strategies to manage episodes of aversive tension or unease. We discuss two recent studies that have found empirical support suggesting that deficits in adequate emotion regulation and distress tolerance play an important role in the development of BPD.

Salsman and Linehan [55•] investigated whether self-reported problems with emotion regulation, as measured by the Difficulties in Emotion Regulation Scale (DERS; [56]), were indirectly associated with self-reported BPD symptoms when negative affect was accounted for. They recruited 456 undergraduates from two geographic locations and found that the 'limited access to emotion regulation strategies' factor was associated with BPD symptoms both when affect intensity and affective reactivity were accounted for. Other studies have found a similar effect for total DERS scores on the association between negative affect on BPD symptoms in both inpatients and undergraduates [51, 57]. These findings support the idea that the inability to appropriately manage affect is an important contributor to BPD.

Extending these findings, Bornovalova, Matusiewicz, and Rojas [58•] examined whether distress tolerance might moderate the relationships of negative emotionality and negative affect intensity on Personality Assessment Inventory-Borderline Features (PAI-BOR; [59]) scores. To measure distress tolerance, they used two laboratory tasks designed to induce frustration and dysphoria, from which a composite score was created. They recruited 110 adult smokers from the community and 76 inpatient substance users. Findings suggested that there was an additive effect for distress tolerance on negative emotionality, such that negative emotionality was associated with PAI-BOR scores regardless of distress tolerance scores, but individuals with the lowest distress tolerance showed the strongest association. However, for affect intensity, there was a relationship with PAI-BOR scores only in individuals with low distress tolerance. Unfortunately, they did not test the cumulative effects of negative emotionality, negative affect intensity, and distress tolerance on BPD traits. Surprisingly, distress tolerance scores were not associated with PAI-BOR scores, contrary to the findings of other studies.

Negative emotionality, defined as a heritable trait reflecting a tendency to experience negative affect, is likely influenced by an emotion sensitivity bias (reviewed above). These findings suggest, then, that the ability to tolerate distress confers some protection against developing BPD symptoms, but more so in individuals who experience intense negative affect than those who are emotionally sensitive. This hints at possibly complex interplay between the different components of emotion dysregulation in individuals with BPD. On that same note, when broken down according to the subscales of the PAI-BOR, findings were only significant for the self-harm subscale, suggesting that individuals who were emotionally sensitive or prone to intense negative affect and who had low distress tolerance scores tended to engage in impulsive and reckless behavior (i.e., maladaptive emotion regulation strategies). 


\section{Maladaptive Regulation Strategies}

The final component of emotion dysregulation is the behavior that may occur instead of appropriate emotion regulation strategies, leading to emotion dysregulation problems that may be observed by others. One explanation of how these behaviors emerge is offered by the emotional cascade model [60]. This model hypothesizes that, if negative affect becomes sufficiently intense, individuals tend to choose maladaptive behaviors over adaptive ones. This may be because maladaptive behaviors often have a more immediate effect or are simpler to employ than more adaptive ones. Yet, although such maladaptive behavior may be effective at reducing negative affect, it is ultimately problematic, either because of negative consequences, because it is not effective over the long-term, or both. Selby et al. [61] found support for this model in individuals with BPD. Similarly, Coifman et al. [47•] found that greater polarity of affect, in the absence of interpersonal stress, predicted increased reports of impulsive and self-injurious behaviors.

There is a great deal of evidence that BPD individuals engage in maladaptive regulation strategies in an attempt to reduce their negative affect. BPD has been linked to maladaptive cognitive strategies such as rumination [61, 62] and thought suppression [63, 64], which often increase, instead of decrease, negative affect. Research also suggests that BPD is associated with experiential avoidance, defined as behavior engaged in to escape an unwanted experience, such as negative affect $[65,66]$. Two types of behaviors that are common in BPD are impulsive and suicidal and self-injurious behaviors. Here, we review the evidence that individuals with BPD engage in impulsive behaviors and suicidal and selfinjurious behavior (SIB) specifically as a means of affect regulation.

In general, BPD is associated with a number of impulsive behaviors that may serve to reduce negative affect, including eating disorders, impulsive buying, and substance use, for example. BPD is also strongly associated with the Urgency subscale of the UPPS Impulsivity Scale [67], although it is associated with other subscales of impulsivity, as well [68]. The Urgency subscale is closely linked to negative affect and refers to the tendency for individuals to act impulsively while in a negative mood, potentially in an attempt to relieve or reduce their negative affect. Tragesser and Robinson [69] found that self-reported affective instability and urgency, as well as (lack of) premeditation, uniquely predicted scores on the PAI-BOR in a sample of undergraduates. From a neurobiological perspective, Silbersweig et al. [70] found that BPD individuals showed decreased activation in the ventromedial prefrontal cortex, relative to controls, in an emotional linguistic go/no-go task requiring behavioral inhibition, but only on trials involving negatively-valenced words. This suggests that this region of the brain might play a role in the tendency for individuals with BPD to act impulsively in the context of negative affect. Conversely, Chapman, Leung, and Lynch [49] found that BPD individuals in a negative emotional state committed fewer impulsive responses on a passive avoidance learning task than BPD individuals not in a negative emotional state. However, this may have been because of the nature of the task, in which stimuli were associated with either reward or punishment and participants had to learn to respond or inhibit responding accordingly. It is difficult to see how acting impulsively on such a task would be tied to negative affect, thereby giving BPD individuals no incentive to act impulsively.

Regarding suicidality, Yen et al. [29] found that affective instability, along with identity disturbance and impulsivity, predicted suicidal behaviors and, with childhood sexual abuse, was a predictor of suicide attempts. There is also evidence that SIB serves as a method of emotion regulation in BPD. SIB is defined as behavior that intentionally damages the body without lethal intent and includes a range of actions (e.g., cutting, burning, scratching). Between 50 and $90 \%$ of individuals with BPD engage in SIB [71, 72]. By far the most 
common reason individuals with BPD give when asked why they engage in SIB is that it reduces feelings of negative affect and helps regulate their mood [73].

Interestingly, individuals with BPD report little or no pain when engaging in SIB or during pain-induction tasks [74-80]. This effect appears to be even greater when participants are experiencing distress $[74,75]$. Franklin and colleagues [81] found that emotion dysregulation mediated the relationship between SIB and pain tolerance, although they did not use a BPD sample. Recently, Niedtfeld et al. [82•] attempted to unravel the neurobiological link between pain and emotion regulation in BPD. Reanalyzing inconclusive data from Niedtfeld et al. [77], they examined the functional connectivity between paralimbic and prefrontal brain structures in individuals with BPD after painful stimulation. Following negative stimulus pictures, BPD patients showed a negative coupling between paralimbic and prefrontal regions, possibly indicating that prefrontal areas inhibited paralimbic regions following pain in this group. Controls showed this same pattern, but only following neutral pictures.

\section{Conclusion}

Having reviewed the four principal components of emotion dysregulation in BPD, as defined by Linehan's biosocial model $[4,5 \bullet]$, it is clear that there are multiple ways of investigating the topic of emotion dysregulation. The available evidence supports the importance of each of these components in BPD. From our review, we can conclude that evidence supports a negative bias in identifying emotions in others and presumably in evaluating others. Such a bias is likely to lead to more intense negative affect in those with $\mathrm{BPD}$, and, in particular, affective instability and reactivity to both events and to others that are encountered in day-to-day life. Unfortunately, those with BPD are relatively unable to tolerate the distress that is encountered in daily life, and they are prone to engage in maladaptive strategies to manage the distress and episodes of intense negative affect (e.g., rumination, thought suppression, experiential avoidance, impulsive behaviors). In summary, our review indicates how pervasive emotion dysregulation is in BPD, manifesting in multiple and distinctive ways.

Unfortunately, many researchers invoke 'emotion dysregulation' without mention of specific components or otherwise clearly defining their use of the term. This can make it difficult to put different studies into context, as they work from different starting points, employ different methods, obtain different outcomes, and yet all purport to study emotion dysregulation. For example, we have demonstrated that sensitivity to emotional stimuli (in this case a negative interpretation bias) and labile negative affect are two distinguishable constructs, but the terms 'emotion sensitivity' and 'affective instability' are both often considered as synonymous and interchangeable with 'emotion dysregulation.'

This practice is short on nuance and has negative consequences for the field. First, it threatens to render the term 'emotion dysregulation' vague and perhaps meaningless. To say that BPD is associated with emotion dysregulation is similar to saying that individuals with BPD have problems with their emotions. This puts those with BPD in the same category as individuals with depression, anxiety, bipolar disorder, schizophrenia, autism spectrum disorder, and even many others who do not meet criteria for a psychiatric disorder. Conversely, to say that BPD is associated with an emotion dysregulation process that consists of emotion sensitivity, heightened negative affect, a deficit of appropriate emotion regulation strategies, and a surplus of inappropriate regulation strategies more precisely specifies the emotion dysregulation problems of individuals with BPD. 
That is not to say that the model provided here is the best or even penultimate model of emotion dysregulation in BPD, or that it is necessarily complete. There may be additional areas of emotion dysregulation that BPD individuals struggle with or, perhaps, some researchers might differentiate between constructs we included within the same component. For example, emotional awareness could be considered distinct from emotion regulation skills. Our decisions on how to define each component are admittedly somewhat subjective, and the full model itself has not been tested empirically. The important takeaway point, however, is that, as research on emotion dysregulation is already being conducted at the level of its components, there is much to be gained by speaking about emotion dysregulation in a more precise manner and as a dynamic process.

Second, defining emotion dysregulation in vague terms likely obscures the interrelationships between the different components of emotion dysregulation. Although some have investigated the relationship between multiple components (e.g., the emotional cascade model proposes a mechanism for how heightened negative affect leads to maladaptive regulation strategies), most studies have focused on only one component. A more precise and dynamic view of emotion dysregulation brings to the forefront questions about how problems in each component develop over time and how emotion dysregulation in BPD is different from emotion dysregulation in other disorders. Research is needed that assesses multiple emotion dysregulation components within the same sample in order to understand how they interact. Many of the assumptions of the biosocial model, and those implicit in our model, remain to be put to an empirical test. For example, our model suggests that earlier components in the model have a causal effect on later ones, but it is possible, perhaps even likely, that different components are mutually reinforcing and that their relationships are more complex.

In summary, emotion dysregulation is a complex process and one that appears to be core to the disorder of BPD. It is also a process that develops over the lifetime, likely beginning in infancy. We have proposed an organizational structure of the components of emotion dysregulation, derived from Linehan's biosocial theory [4, 5•]: Emotion sensitivity, heightened negative affect, a deficit of appropriate regulation strategies, and a surplus of maladaptive regulation strategies. We believe that there are important benefits for research that first specifies those aspects of emotion dysregulation under study and, second, examines these components in the context of other emotion dysregulation components.

\section{References}

Papers of particular interest, published recently, have been highlighted as:

- Of importance,

•• Of major importance

1. Lenzenweger MF, Lane MC, Loranger AW, Kessler RC. DSM-IV personality disorders in the national comorbidity survey replication. Biol Psychiatry. 2007; 62:553-64. [PubMed: 17217923]

2•. Trull TJ, Jahng S, Tomko RL, et al. Revised NESARC personality disorder diagnoses: gender, prevalence, and comorbidity with substance dependence disorders. J Pers Disord. 2010; 24:41226. This article provides the most recent epidemiologic data on PDs in the United States. [PubMed: 20695803]

3. Widiger, TA.; Trull, TJ. Borderline and narcissistic personality disorders. In: Sutker, P.; Adam, H., editors. Comprehensive Textbook of Psychopathology. New York: Plenum; 1993. p. 371-94.

4. Linehan, MM. Cognitive-Behavioral Treatment of Borderline Personality Disorder. New York: Guilford; 1993. 
5•. Crowell SE, Beauchaine TP, Linehan MM. A biosocial developmental model of borderline personality: elaborating and extending Linehan's theory. Psychol Bull. 2009; 135:495-510. This article provides the most recent version of Linehan's biosocial theory of BPD. [PubMed: 19379027]

6. Millon, T. Disorders of the personality: DSM-IV and beyond. New York: Wiley; 1996.

7. Westen D, Muderrisoglu S, Fowler C, et al. Affect regulation and affective experience: Individual differences, group differences, and measurement using a Q-sort procedure. J Consult Clin Psychol. 1997; 65:429-39. [PubMed: 9170766]

8. Werner, K.; Gross, JJ. Emotion regulation and psychopathology: A conceptual framework. In: Kring, AM.; Sloan, DM., editors. Emotion regulation and psychopathology: A transdiagnostic approach to etiology and treatment. New York: Guilford Press; 2010. p. 13-37.

9. Sieswerda S, Arntz A, Kindt M. Successful Psychotherapy Reduces Hypervigilance in Borderline Personality Disorder. Behav Cogn Psychother. 2007; 35:387-402.

10. Sieswerda S, Arntz A, Mertens I, Vertommen S. Hypervigilance in patients with borderline personality disorder: Specificity, automaticity, and predictors. Behav Res Ther. 2007; 45:1011-24. [PubMed: 17045566]

11. Carlson EA, Egeland B, Sroufe LA. A prospective investigation of the development of borderline personality symptoms. Dev Psychopathol. 2009; 21:1311-34. [PubMed: 19825270]

12. Stroop JR. Studies of interference in serial verbal reactions. J Exp Psychol Gen. 1935; 18:643-62.

13. Arntz A, Appels C, Sieswerda S. Hypervigilance in borderline disorder: A test with the emotional Stroop paradigm. J Pers Disord. 2000; 14:366-73. [PubMed: 11204344]

14. Domes G, Winter B, Schnell K, et al. The influence of emotions on inhibitory functioning in borderline personality disorder. Psychol Med. 2006; 36:1163-72. [PubMed: 16700964]

15. Sprock J, Rader TJ, Kendall JP, Yoder CY. Neuropsychological functioning in patients with borderline personality disorder. J Clin Psychol. 2000; 56:1587-600. [PubMed: 11132573]

16. Wingenfeld K, Rullkoetter N, Mensebach C, et al. Neural correlates of the individual emotional Stroop in borderline personality disorder. Psychoneuroendocrinology. 2009; 34:571-86. [PubMed: 19042093]

17. Domes G, Schulze L, Herpertz SC. Emotion recognition in borderline personality disorder-a review of the literature. J Pers Disord. 2009; 23:6-19. [PubMed: 19267658]

18. Domes G, Czieschnek D, Weidler F, Berger C, Fast K, Herpertz SC. Recognition of facial affect in borderline personality disorder. J Pers Disord. 2008; 22:135-47. [PubMed: 18419234]

19. Scott LN, Levy KN, Adams RB, Stevenson MT. Mental state decoding abilities in young adults with borderline personality disorder traits. Pers Disord Theory Res Treat. 2011; 2:98-112.

20. Wagner AW, Linehan MM. Facial expression recognition ability among women with borderline personality disorder: Implications for emotion regulation? J Pers Disord. 1999; 13:329-44. [PubMed: 10633314]

21. Koenigsberg HW, Siever LJ, Lee H, et al. Neural correlates of emotion processing in borderline personality disorder. Psychiatry Res. 2009; 172:192-9. [PubMed: 19394205]

22. Bland AR, Williams CA, Scharer K, Manning S. Emotion processing in borderline personality disorders. Issues Ment Health Nurs. 2004; 25:655-72. [PubMed: 15371135]

23. Levine D, Marziali E, Hood J. Emotion processing in borderline personality disorders. J Nerv Ment Dis. 1997; 185:240-6. [PubMed: 9114809]

24. Minzenberg MJ, Fan J, New AS, et al. Fronto-limbic dysfunction in response to facial emotion in borderline personality disorder: An event-related fMRI study. Psychiatry Res. 2007; 155:231-43. [PubMed: 17601709]

25. Guitart-Masip M, Pascual JC, Carmona S, et al. Neural correlates of impaired emotional discrimination in borderline personality disorder: an fMRI study. Prog Neuropsychopharmaco Biol Psychiatry. 2009; 33:1537-45.

26. Mauchnik J, Schmahl C. The latest neuroimaging findings in borderline personality disorder. Curr Psychiatry Rep. 2010; 12:46-55. [PubMed: 20425310]

27. Koenigsberg HW, Harvey PD, Mitropoulou V, et al. Characterizing Affective Instability in Borderline Personality Disorder. Am J Psychiatry. 2002; 159:784-8. [PubMed: 11986132] 
28. Trull TJ. Structural relations between borderline personality disorder features and putative etiological correlates. J Abnorm Psychol. 2001; 110:471-81. [PubMed: 11502090]

29. Yen S, Zlotnick C, Costello E. Affect regulation in women with borderline personality disorder traits. J Nerv Ment Dis. 2002; 190:693-6. [PubMed: 12409863]

30. Kuo JR, Linehan MM. Disentangling emotion processes in borderline personality disorder: physiological and self-reported assessment of biological vulnerability, baseline intensity, and reactivity to emotionally evocative stimuli. J Abnorm Psychol. 2009; 118:531-44. [PubMed: 19685950]

31 . Nica EI, Links PS. Affective instability in borderline personality disorder: experience sampling findings. Curr Psychiatry Rep. 2009; 11:74-81. This article reviews EMA findings on affective instability in BPD. [PubMed: 19187713]

32. Henry C, Mitropoulou V, New AS, et al. Affective instability and impulsivity in borderline personality and bipolar II disorders: similarities and differences. J Psychiatric Res. 2001; 35:30712.

33. Trull T, Solhan M, Tragesser SL, et al. Affective instability: Measuring a core feature of borderline personality disorder with ecological momentary assessment. J Abnorm Psychol. 2008; 117:64761. [PubMed: 18729616]

34॰. Ebner-Priemer UW, Eid M, Stabenow S, et al. Analytic strategies for understanding affective (in)stability and other dynamic processes in psychopathology. J Abnorm Psychol. 2009; 118:195-202. This article outlines important considerations for measuring affective instability using EMA. [PubMed: 19222325]

35. Stone AA, Shiffman S. Ecological momentary assessment (EMA) in behavioral medicine. Ann Behav Med. 1994; 16:199-202.

36. Trull TJ, Ebner-Priemer UW. Using Experience Sampling Methods/Ecological Momentary Assessment (ESM/EMA) in Clinical Assessment and Clinical Research: Introduction to the Special Section. Psychol Assess. 2009; 21:457-62. [PubMed: 19947780]

37. Walls, TA.; Schafer, JL. Models for intensive longitudinal data. New York: Oxford University Press; 2006.

38. Solhan M, Trull TJ, Jahng S, Wood P. Clinical assessment of affective instability: comparing EMA indices, questionnaire reports, and retrospective recall. Psychol Assess. 2009; 21:425-36. [PubMed: 19719353]

39. Shiffman S, Stone AA, Hufford MR. Ecological momentary assessment. Annu Rev Clin Psycho. 2008; 4:1-32.

40. Ebner-Priemer UW, Kuo J, Kleindienst N, et al. State affective instability in borderline personality disorder assessed by ambulatory monitoring. Psychol Med. 2007; 37:961-70. [PubMed: 17202005]

41. Larsen R. The stability of mood variability: a spectral analytic approach to daily mood assessments. J Pers Soc Psychol. 1987; 52:1195-204.

42. Gross JJ. The emerging field of emotion regulation: an integrative review. Rev Gen Psychol. 1998; 2:271-99.

43. Gross JJ, John OP. Individual differences in two emotion regulation processes: Implications for affect, relationships, and well-being. J Pers Soc Psychol. 2003; 85:348-62. [PubMed: 12916575]

44. Leible TL, Snell WE. Borderline personality disorder and multiple aspects of emotional intelligence. Personal Individ Differ. 2004; 37:393-404.

45. Barrett LF. Feelings or words? Understanding the content in self-report ratings of experienced emotion. J Pers Soc Psychol. 2004; 87:266-81. [PubMed: 15301632]

46•. Suvak MK, Litz BT, Sloan DM, et al. Emotional granularity and borderline personality disorder. J Abnorm Psychol. 2011; 120:414-26. Empirical investigation of emotional granularity in BPD. Found that BPD individuals emphasized valence more and arousal less than controls. [PubMed: 21171723]

47•. Coifman KG, Berenson KR, Rafaeli E, Downey G. From Negative to Positive and Back Again: Polarized Affective and Relational Experience in Borderline Personality Disorder. J Abnorm Psychol. 2012; 121:668-79. EMA investigation of emotion polarity in BPD. Found that individuals with BPD experienced more polarity than controls. [PubMed: 22686872] 
48. Bornovalova MA, Gratz KL, Daughters SB, et al. A multimodal assessment of the relationship between emotion dysregulation and borderline personality disorder among inner-city substance users in residential treatment. J Psychiatric Res. 2008; 42:717-26.

49. Chapman AL, Leung DW, Lynch TR. Impulsivity and emotion dysregulation in borderline personality disorder. J Pers Disord. 2008; 22:148-64. [PubMed: 18419235]

50. Gratz KL, Rosenthal MZ, Tull MT, Lejuez CW, Gunderson JG. An experimental investigation of emotion dysregulation in borderline personality disorder. J Abnorm Psychol. 2006; 115:850-5. [PubMed: 17100543]

51. Gratz KL, Tull MT, Baruch DE, Bornovalova MA, Lejuez CW. Factors associated with cooccurring borderline personality disorder among inner-city substance users: the roles of childhood maltreatment, negative affect intensity/reactivity, and emotion dysregulation. Compr Psychiatry. 2008; 49:603-15. [PubMed: 18970909]

52. Koenigsberg HW, Fan J, Ochsner KN, et al. Neural correlates of the use of psychological distancing to regulate responses to negative social cues: a study of patients with borderline personality disorder. Biol Psychiatry. 2009; 66:854-63. [PubMed: 19651401]

53. Schulze L, Domes G, Kruger A, et al. Neuronal correlates of cognitive reappraisal in borderline patients with affective instability. Biol Psychiatry. 2011; 69:564-73. [PubMed: 21195392]

54. Leyro T, Zvolensky M, Bernstein A. Distress tolerance and psychopathological symptoms and disorders: a review of the empirical literature among adults. Psychol Bull. 2010; 136:576-600. [PubMed: 20565169]

55•. Salsman NL, Linehan MM. An Investigation of the Relationships among Negative Affect, Difficulties in Emotion Regulation, and Features of Borderline Personality Disorder. J Psychopathol Behav Assess. 2012; 34:260-7. Found that deficits in emotion regulation were associated with BPD symptoms in two models, one controlling for negative affective intensity, and one controlling for negative affective reactivity.

56. Gratz KL, Roemer L. Multidimensional assessment of emotion regulation and dysregulation: Development, factor structure, and initial validation of the difficulties in emotion regulation scale. J Psychopathol Behav Assess. 2004; 26:41-54.

57. Glenn CR, Klonsky ED. Emotion dysregulation as a core feature of borderline personality disorder. J Pers Disord. 2009; 23:20-8. [PubMed: 19267659]

58•. Bornovalova MA, Matusiewicz A, Rojas E. Distress tolerance moderates the relationship between negative affect intensity with borderline personality disorder levels. Compr Psychiatry. 2011; 52:744-53. Found that negative emotionality was associated with BPD symptoms regardless of distress tolerance scores, while affective intensity was associated with BPD symptoms only in individuals with low distress tolerance. [PubMed: 21257162]

59. Morey, LC. Personality Assessment Inventory: Professional manual. Odessa: Psychological Assessment Resources; 1991.

60. Selby EA, Anestis MD, Joiner TE. Understanding the relationship between emotional and behavioral dysregulation: Emotional cascades. Behav Res Ther. 2008; 46:593-611. [PubMed: 18353278]

61. Selby EA, Anestis MD, Bender TW, Joiner TE. An exploration of the emotional cascade model in borderline personality disorder. J Abnorm Psychol. 2009; 118:375-87. [PubMed: 19413411]

62. Baer RA, Sauer SE. Relationships between depressive rumination, anger rumination, and borderline personality features. Pers Disord Theory Res Treat. 2011; 2:142-50.

63. Cheavens JS, Rosenthal MZ, Daughters SB, et al. An analogue investigation of the relationships among perceived parental criticism, negative affect, and borderline personality disorder features: The role of thought suppression. Behav Res Ther. 2005; 43:257-68. [PubMed: 15629754]

64. Rosenthal ZM, Cheavens JS, Lejuez CW, Lynch TR. Thought suppression mediates the relationship between negative affect and borderline personality disorder symptoms. Behav Res Ther. 2005; 43:1173-85. [PubMed: 16005704]

65. Chapman AL, Dixon-Gordon KL, Walters KN. Experiential Avoidance and Emotion Regulation in Borderline Personality Disorder. J Ration Emot Cogn Behav Ther. 2011; 29:35-52. 
66. Hayes SC, Wilson KG, Gifford EV, Follette VM, Strosahl K. Experiential avoidance and behavioral disorders: A functional dimensional approach to diagnosis and treatment. J Consult Clin Psychol. 1996; 64:1152-68. [PubMed: 8991302]

67. Whiteside SP, Lynam DR. The Five Factor Model and impulsivity: Using a structural model of personality to understand impulsivity. Personal Individ Differ. 2001; 30:669-89.

68. Whiteside SP, Lynam DR, Miller JD, Reynolds SK. Validation of the UPPS impulsive behaviour scale: A four-factor model of impulsivity. Eur J Pers. 2005; 19:559-74.

69. Tragesser SL, Robinson RJ. The role of affective instability and UPPS impulsivity in borderline personality disorder features. J Pers Disord. 2009; 23:370-83. [PubMed: 19663657]

70. Silbersweig D, Clarkin JF, Goldstein M, et al. Failure of frontolimbic inhibitory function in the context of negative emotion in borderline personality disorder. Am J Psychiatry. 2007; 164:183241. [PubMed: 18056238]

71. Dulit RA, Fyer MR, Leon AC, et al. Clinical correlates of self-mutilation in borderline personality disorder. Am J Psychiatry. 1994; 151:1305. [PubMed: 8067485]

72. Zanarini MC, Frankenburg FR, Reich DB, et al. The 10-year course of physically self-destructive acts reported by borderline patients and axis ii comparison subjects. Acta Psychiatr Scand. 2008; 117:177-84. [PubMed: 18241308]

73. Klonsky ED. The functions of deliberate self-injury: a review of the evidence. Clin Psychol Rev. 2007; 27:226-39. [PubMed: 17014942]

74. Bohus M, Limberger M, Ebner U, et al. Pain perception during self-reported distress and calmness in patients with borderline personality disorder and self-mutilating behavior. Psychiatry Res. 2000; 95:251-60. [PubMed: 10974364]

75. Ludäscher P, Bohus M, Lieb K, et al. Elevated pain thresholds correlate with dissociation and aversive arousal in patients with borderline personality disorder. Psychiatry Res. 2007; 149:291-6. [PubMed: 17126914]

76. McCown W, Galina H, Johnson J, et al. Borderline personality disorder and laboratory-induced cold pressor pain: evidence of stress-induced analgesia. J Psychopathol Behav Assess. 1993; 15:87-95.

77. Niedtfeld I, Schulze L, Kirsch P, et al. Affect regulation and pain in borderline personality disorder: A possible link to the understanding of self-injury. Biol Psychiatry. 2010; 68:383-91. [PubMed: 20537612]

78. Russ MJ, Roth SD, Lerman A, et al. Pain perception in self-injurious patients with borderline personality disorder. Biol Psychiatry. 1992; 32:501-11. [PubMed: 1445967]

79. Schmahl C, Greffrath W, Baumgartner U, et al. Differential nociceptive deficits in patients with borderline personality disorder and self-injurious behavior: Laser-evoked potentials, spatial discrimination of noxious stimuli, and pain ratings. Pain. 2004; 110:470-9. [PubMed: 15275800]

80. Schmahl C, Bohus M, Esposito F, et al. Neural correlates of antinociception in borderline personality disorder. Arch Gen Psychiatry. 2006; 63:659-67. [PubMed: 16754839]

81. Franklin JC, Aaron RV, Arthur MS, Shorkey SP, Prinstein MJ. Nonsuicidal self-injury and diminished pain perception: the role of emotion dysregulation. Compr Psychiatry. 2012; 53:691700. [PubMed: 22208846]

82. Niedtfeld I, Kirsch P, Schulze L, et al. Functional connectivity of pain-mediated affect regulation in Borderline Personality Disorder. PLoS One. 2012; 7:e33293. Found a negative coupling between paralimbic and frontal regions in the brain of BPD individuals following painful stimulus and presentation of a negatively valenced picture. [PubMed: 22428013] 


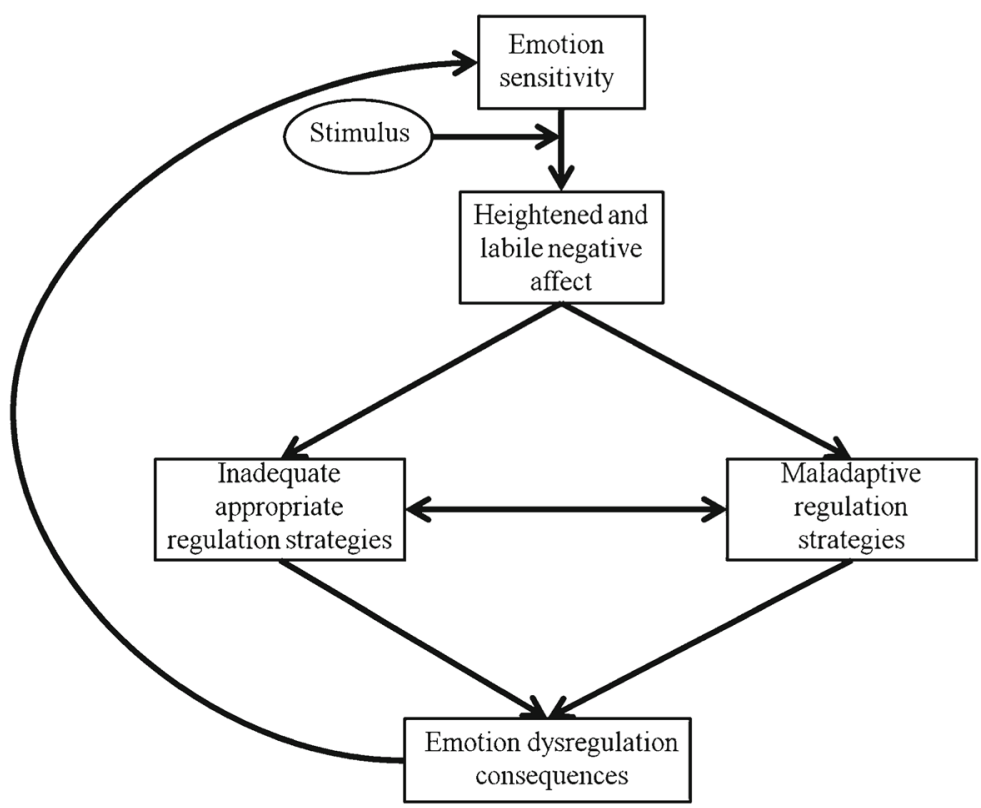

Fig. 1.

Multi-component model of emotion dysregulation in BPD. Individuals with BPD are theorized to be sensitive to emotional stimuli from birth. Experiencing a negatively valenced stimulus (or interpreting a stimulus in a negative way) in the environment leads to increases in negative affect and affective instability. Heightened and unstable negative affect both makes it difficult to learn and to employ appropriate emotion regulation strategies and leads to an increase in maladaptive and impulsive regulation strategies. Emotion dysregulation consequences occur as a result, which, in turn, reinforce vigilance toward negatively valenced stimuli in the environment 